\title{
Palliative care: needs of advanced breast cancer patients
}

This article was published in the following Dove Press journal:

Breast Cancer - Targets and Therapy

\author{
Nathan I Cherny \\ Shani Paluch-Shimon \\ Yael Berner-Wygoda \\ Integrated Oncology and Palliative \\ Medicine Department, Breast \\ Oncology Unit, Shaare Zedek Medical \\ Center, Jerusalem, Israel
}

\begin{abstract}
Advanced breast cancer is characterized by many physical manifestations with the potential to undermine the quality of life (most related to the cancer and some to treatments), as well as substantial impact on psychosocial well-being. Patients with advanced breast cancer and their families have complex needs that have to be addressed in order to minimize severe distress and deterioration in the quality of life of patients and their family members. This task requires the full engagement of an interdisciplinary approach to palliative care with strong emphasis on the assessment of needs and anticipated needs, patient expectations, skilled therapeutics, and commitment to continuity of care. In this review, we address four issues: 1) organizational and conceptual issues in palliative care of patients with breast cancer, 2) common physical symptoms among patients with breast cancer and their management, 3) common psychological issues among patients with breast cancer, and 4) common challenging palliative care problems in breast cancer. Keywords: psychooncology, supportive care, goal setting, quality of life
\end{abstract}

\section{Introduction}

Breast cancer is the most common cancer in women and is the leading cause of cancerrelated death in women in both developing and developed regions. Approximately $5 \%-10 \%$ of newly diagnosed patients with breast cancer are metastatic at diagnosis; of these, approximately one-fifth will survive for 5 years. Among patients diagnosed with early stage disease, depending on the risk factors, as many as $30 \%$ of node-negative and up to $70 \%$ of node-positive breast cancers will develop metastatic disease. ${ }^{1,2}$

Since the survival of women with metastatic breast cancer is often prolonged, the prevalence of metastatic disease is high, and indeed, the care for women with metastatic breast cancer is a major challenge for oncologists and palliative care teams. ${ }^{1}$ The natural history of breast cancer is variable - those with aggressive subtypes, such as triple negative breast cancer, may have very fulminant courses leading to death within a few months or years while those with hormone receptor-positive and Her-2-overexpressed metastatic breast cancer will have a prolonged course of illness. ${ }^{1}$

Breast cancer is characterized by many physical manifestations with the potential to undermine the quality of life (most related to the cancer and some to treatments), as well as substantial impact on psychosocial well-being. ${ }^{2,3}$ Antineoplastic treatment with hormonal therapy, chemotherapy, and biological therapies can modify and relieve physical manifestations of the disease, and indeed, besides their impact on prolongation of survival, these treatments are a cornerstone of palliation for women with advanced breast cancer.

With the passage to time and sequential lines of treatment, the therapeutic window narrows down and the likelihood of achieving substantial benefit from disease-modifying
Correspondence: Nathan I Cherny Integrated Oncology and Palliative Medicine Department, Department, Breast Oncology Unit, Shaare Zedek Medical Center, Shmu'el Bait St I2, Jerusalem, 9103102, Israel Email Chernyn@netvision.net.il 
therapies is diminished until such a time that further trials of disease-modifying treatments are either no longer helpful or have greater likelihood of harm than benefit, at which point palliation and support become the central focus of care.

All of this is reflected in the goals of care, whereas for patients with early metastatic cancer, the goal of prolongation of survival may sometimes even take precedence over considerations of comfort; as the disease progresses and the likelihood of life prolongation diminishes and issues of comfort and function take greater precedence. In all cases, the prevailing goals of care need to be discussed with the patient and their family, and all stakeholders should be aware of the prevailing priorities since these set the context for decisionmaking and care planning. ${ }^{4}$

A complete review of all the aspects of palliative care for cancer patients is beyond the scope of what can be reviewed in a single paper. In this review, we will focus on four issues: 1) organizational and conceptual issues in palliative care of patients with breast cancer, 2) common physical symptoms among patients with breast cancer and their management, 3) common psychological issues among patients with breast cancer, and 4) common challenging palliative care problems in breast cancer.

\section{Organizational and conceptual issues in palliative care of patients with breast cancer Models of care for patients with advanced breast cancer}

The high prevalence of physical and psychosocial distress among patients with breast cancer underscores the need for an approach to care that incorporates clinical approach to address these issues in parallel to usual anticancer therapies.

There is no one best way for oncologists and specialist palliative care services to work together. Rather a number of different models have been developed. ${ }^{5}$

\section{Oncologist-based palliative care}

In this model, the oncologists assume the role of coordinating care and providing both anticancer and palliative care services, thus seeing the patients through from diagnosis until death. This approach emphasizes the importance of the oncologist-patient relationship and the notions of continuity of care and nonabandonment. The success of this model is dependent on the level of palliative care skills and sophistication of the oncologists and their ability to balance competing intellectual and practical interests. This approach is augmented by having a strong relationship with a care team, including a palliative medicine expert for backup in difficult cases.

\section{Concurrent model}

In this model, patients with advanced cancer are jointly cared for by both an oncologist and a palliative medicine specialist (working in the context of an interdisciplinary palliative care team) in a similar way in which medical, surgical, and radiation oncologists traditionally work together. ${ }^{6}$ The relative role of the oncologist or palliative medicine physician is determined by the prevailing problems, and indeed, case management responsibilities may be handled by either and are usually determined by the circumstances of the patient. Successful implementation of this approach requires close cooperation and open communication between the oncology and palliative medicine clinicians. This approach is strongly supported by data demonstrating improved patient outcomes. $^{7-12}$

\section{Assessing patient needs for palliative care}

An appreciation of the complete diversity of factors that may contribute to patient distress underscores the need for a methodical approach to the assessment of each individual case. ${ }^{13}$ This role should be assumed by a case manager, who in many cases is both the clinician and the coordinator of the health care professionals, that is, physicians, nurses, social workers, and therapists. The evaluation must incorporate the following.

\section{Patient assessment}

The early establishment of good symptom control conveys concern, builds the trust of patients and their families, and facilitates the ability to address other important issues.

Patient variables that must be assessed include the disease status, expected disease progression, present functional level, symptoms, current therapies, and anticipated future problems. Of particular importance is the patients' level of function, reflecting their mobility, ability to communicate, ability to perform activities of daily living, bowel and bladder function, and level of alertness. The use of validated pain and symptom assessment instruments can provide a format for communication between the patients and the health care professionals, and these instruments can also be used to monitor the adequacy of therapy.

It is important to ascertain the patients' and their families' understanding of the nature and extent of the illness and their expectations of treatment and outcome. As a part of this process, the case manager must develop an understanding of the patients' prioritization of the sometimes conflicting 
goals of care: optimization of comfort, function (interactional function in particular), and duration of survival.

\section{Family assessment}

Family assessment should encompass medical variables, psychosocial concerns, and the adequacy and availability of supports. Evaluation of the willingness and ability of home carers to provide home care, and the availability of supports are essential. Concurrent medical problems in a family member, particularly a primary caregiver, need to be evaluated since the viability of the home care plan may depend upon the family members' ability to participate in care. Since the ability of families to cope with home care is largely determined by the nature of the available home care supports, family assessment must include an assessment of available health care professional and community supports.

Discrepancies between what is known and understood by the patients and their families should be identified, and the reasons for these discrepancies should be tactfully explored. Knowledge deficits may have been deliberately maintained: the family or patient not wanting information overload, the patient protecting the family from the knowledge of poor prognosis, or the family protecting the patient from the impact of such information. ${ }^{14}$

\section{Health care professional assessment}

To effectively plan for ongoing care, the case manager must understand the limitations of the involved health care professionals (knowledge, experience, and availability for home care), their difficulties in coping with the situation, and their perceived needs to improve the care outcome.

Based on this assessment, one can formulate a care plan that addresses all aspects of the care trilogy: patient, family, and health care professionals. The formulation can be summarized in a document or report that describes:

1. The medical condition of the patient and the goals of care

2. Description of the involved family members and health care professionals

3. Patient issues: physical, psychological, existential, social, communication, understanding

4. Family issues: physical, psychological, existential, social, communication, understanding

5. Professional carer issues: staffing, training, resources, resource/need match, emotional coping

6. Coping assessment: patient, family, health care professionals

7. Contingency planning: anticipated contingencies, planned interventions
Since care for the palliative care patients is a longitudinal commitment, assessment must be repeated at appropriate intervals which will be determined by the rate of change in the patients' clinical conditions or at points of major change in goals, care plan, or the patients' conditions.

\section{Family meetings and goals of care}

Family meetings, with relevant members of the professional health care team, provide a useful format for discussing the needs of all parties involved, clarifying care goals, sharing and exploring concerns, and developing a therapeutic plan that adequately addresses those needs. ${ }^{15,16}$ The participants should be determined on an individual case basis.

Since family is an appropriate unit of care, and its members have a right to confidentiality, it may be appropriate, occasionally, to meet without the participation of the patient to address their concerns and needs. Meetings with the participation of all persons who are involved can open communication, improve coordination in the formulation of a care plan, and facilitate better personal coping for each of the individuals involved.

The case manager is responsible for monitoring the degree to which care needs are being met and for facilitating change when necessary. Similarly the well-being and function of the health care professionals must be monitored, ensuring the availability of appropriate manpower and expertise to effectively manage the prevailing problems. For security and safety in the event of a clinical crisis, it is essential that the patients and their families have access to a contact person with 24 hour availability. This model represents a family cantered, multidisciplinary, collaborative approach between physicians, nurses, social workers, other therapists, and community supports.

\section{Evaluation and management of common physical symptoms Pain}

Overwhelmingly, the most common causes of cancer-related pain in women with breast cancer are bone metastases and their sequelae including impending and actual fractures and compression of adjacent neural structures. ${ }^{17,18}$ Other pain syndromes that are less common include tumor pain from chest wall infiltration, brachial plexopathy, headache from brain metastases or leptomeningeal metastases, and abdominal pain from hepatic capsular distension. ${ }^{17}$

Bone metastases are very common in breast cancer. ${ }^{18}$ They most commonly involve the axial skeleton, ${ }^{19}$ these lesions may be either blastic or lytic; however, when caused 
by bone marrow infiltration, the disease may not be evident on conventional imaging and may require either MRI or PET CT imaging. ${ }^{18}$

The vertebrae are the most common sites of bony metastases. More than two-thirds of vertebral metastases are located in the thoracic spine; lumbosacral and cervical metastases account for $\sim 20 \%$ and $10 \%$, respectively. The early recognition of pain syndromes due to tumor invasion of vertebral bodies is essential, since pain usually precedes compression of adjacent neural structures and prompt treatment of the lesion may prevent the subsequent development of neurologic deficits.

The pelvis and hip are common sites of metastatic involvement. The weight bearing function of these structures, essential for normal ambulation, contributes to the propensity of the disease at these sites to cause incident pain with walking and weight-bearing. ${ }^{20}$

The management of pain requires an integrated approach including antitumor therapies (including disease-modifying agents: hormonal, chemotherapeutic and biological, radiotherapy, and surgery), analgesic therapy, and rehabilitation and psychological care. ${ }^{18,19,21}$ Analgesic pharmacotherapy must be cognoscente of the mechanism of pain (somatic vs neuropathic) and must be appropriate to the severity of the pain. Opioid analgesic therapy will be needed for most patients with strong pain, and this should incorporate the background around the clock dosing for chronic pain as well as provision for as needed "rescue doses" using a rapidly acting opioid. Opioid analgesic therapy must be titrated to effect with close follow-up both for the adequacy of analgesia and for the emergence of adverse effects such as drowsiness, confusion, nausea, itch, myoclonus, or constipation. If either inadequate analgesia or excessive adverse effects are observed, the treatment strategy will need revision until adequate and acceptable balance is found between the provision of relief and adverse effects. When the mechanism of the pain is neuropathic, patients may benefit from the addition of an agent with specific efficacy for this mechanism of pain such as a tricyclic, an SNRI, and/or an anticonvulsant. Radiotherapy may be particularly important for patients with poorly controlled pain from bone metastases and for patients with compression or infiltration of neural structures.

\section{The special case of back pain and epidural compression}

Epidural compression of the spinal cord or cauda equina is a common neurologic complication of breast cancer. Most often it is caused by posterior extension of vertebral body metastasis to the epidural space. Occasionally, epidural compression is caused by tumor extension from the posterior arch of the vertebra or infiltration of a paravertebral tumor through the intervertebral foramen.

Untreated, epidural compression leads inevitably to neurological damage. Effective treatment can potentially prevent these complications and thus underscores the importance of early diagnosis. Since pain usually precedes neurologic signs by a prolonged period, it should be viewed as a potential indicator of epidural compression, which can lead to treatment at a time when a favorable response is most likely. Some pain characteristics are particularly suggestive of epidural extension: rapid worsening of back pain and radicular pain (particularly if it is exacerbated by recumbence, cough, sneeze, or strain). Weakness, sensory loss, autonomic dysfunction, and reflex abnormalities usually occur after a period of progressive pain.

Patients with suspected epidural encroachment require urgent axial assessment of the epidural space with either MRI or CT imaging. MRI offers accurate imaging of the vertebrae, intraspinal, and paravertebral structures. ${ }^{22,23} \mathrm{CT}$ imaging is a good option if urgent MRI cannot be arranged.

Patients with identified epidural encroachment will need treatment with either radiotherapy with steroids or a combination of surgical compression and stabilization followed by radiotherapy. ${ }^{22,23}$ There is conflicting data as to whether the later yields better neurological outcomes. ${ }^{22,24,25}$

\section{latrogenic chronic pain}

Iatrogenic pain is a major cause of patient distress even among patients with early stage disease. Three iatrogenic pain syndromes are extremely common and a major cause of patient distress; these include: 1) post axillary dissection neuropathic pain, ${ }^{26} 2$ ) taxane-induced painful peripheral neuropathy, ${ }^{26}$ and 3 ) aromatase inhibitor (AI)-induced multifocal joint pains. ${ }^{27}$ The risk of postoperative neuropathy is reduced with the application of more limited dissection approaches such as sentinel lymph node dissection, but even with these approaches, there remain a subgroup of patients who will endure an ongoing neuropathic pain syndrome.

Taxane-induced peripheral neuropathy is a common consequence of breast cancer treatment in both the adjuvant and metastatic settings. ${ }^{28,29}$ The risk of chronic neuropathy must be discussed with all patients considering taxane therapy and must be considered in any risk-benefit evaluation regarding the potential role of these agents. ${ }^{30}$ Treatment of established neuropathy is challenging and often requires an interdisciplinary approach including occupational therapy to assist 
with functional issues, pharmacotherapy for neuropathic pain, ${ }^{31}$ and physiological and social support. Pharmacotherapy approaches should be evidence based and will commonly require sequential trials of therapy to optimal benefit and minimize adverse effects. ${ }^{31}$

AIs can cause arthralgia, joint stiffness, and bone pain, which can be severe in up to $30 \%$ of patients. The pain and stiffness typically involve the hands, arms, knees, feet, pelvic and hip bones, or back. It is usually symmetrical and may be associated with mild soft-tissue thickening. ${ }^{27}$ There is limited evidence to suggest that some patients may benefit from a change to a different AI formulation. There is limited anecdotal evidence support of vitamin D supplementation, nonsteroidal anti-inflammatory medications, yoga, exercise, acupuncture, or SSRI antidepressants. In some cases, this becomes an intractable problem that necessitates a therapeutic compromise including reversion back to tamoxifen.

\section{Breathlessness (dyspnea)}

Dyspnea is defined as a subjective sensation of difficulty in breathing. It is a common symptom among patients with advanced breast cancer. ${ }^{32}$ Among patients with breast cancer, there are many potential causes of breathlessness (Table 1). Since many of these are amenable to specific therapies, optimal treatment requires careful evaluation which must be cognoscente of the patients' premorbid state, current therapy, and known extent of disease.

Except in situations where dyspnea is anticipated because of the extent of disease or in the setting of imminent anticipated dying, dyspnea ought to be carefully evaluated including a careful physical examination, pulse oximetry, and chest X-ray. Other investigations including ECG, echo cardiogram, CT, or CT angiography may be needed, and these should be judiciously selected on the basis of the differential diagnosis suggested by the medical history and physical examination findings.

Symptomatic management of dyspnea uses nonpharmacological and pharmacological approaches. ${ }^{32}$ Sitting the patient upright, increasing air flow over the face using a fan or open window, and use of bedside relaxation techniques are all helpful. Treatable precipitants may benefit from specific therapies. Large symptomatic pleural or pericardial effusions should be drained. Recurrent pleural effusions, particularly in patients with a very refractory disease may require pleuradesis or a permanent drainage device (such as a PleurX catheter). Oxygen supplementation may help relieve dyspnea for patients who are hypoxemic. Most patients will
Table I Potentially treatable causes of breathlessness

\begin{tabular}{|l|l|}
\hline Underlying cause & Potential treatment \\
\hline Anemia & Blood transfusion \\
\hline Acidosis & $\mathrm{HCO}_{3}$ \\
\hline Bronchospasm & Bronchodilator therapy \\
\hline Pneumonia & Antibiotic therapy \\
\hline Pulmonary embolus & Anti-coagulate \\
\hline Pneumonitis & Steroids \\
\hline Atrial fibrillation & Anti-arrhythmic \\
\hline Congestive cardiac failure & Diuretic \\
\hline Pericardial tamponade & Drainage \\
\hline Pleural effusion & Thoracentesis \\
\hline Endobronchial tumor & Endobronchial laser \\
\hline Extrinsic compression of bronchus & Radiotherapy \\
\hline Carcinomatous lymphangitis & Steroids (anecdotes only) \\
\hline Lung metastases & Chemotherapy/hormonal therapy \\
\hline
\end{tabular}

be adequately assisted by nasal prongs or a face mask. Some patients may benefit from either BiPAP or high-flow oxygen to maintain adequate oxygenation and comfort. Patients with severe hypoxemia that is caused by a potentially reversible acute problem such as pneumonia or pneumonitis may require ventilatory support to enable them to overcome an acute episode. Consequently, it is helpful to evaluate patients' advanced directives should they have an acute life-threatening but potentially reversible event.

Opioids are the preferred symptomatic therapy for dyspnea at the end-of-life as well as dyspnea that is not adequately relieved by the treatment of the underlying cause and correction of hypoxemia. In the opioid naïve patients, low doses of oral $(5-10 \mathrm{mg})$ or parenteral morphine (2-4 $\mathrm{mg}$ ) may be adequate for most of the patients. Importantly, patients on chronic opioid therapy may need higher doses. Continual opioid therapy can be provided using long-acting oral or transdermal formulations or by continuous infusion. Patients with severe dyspnea should also have access to a rescue dose analogous to the treatment of breakthrough pain.

\section{Fatigue}

Fatigue is one of the most common symptoms among patients with breast cancer. It is a major cause of distress to both patients and their family members. It has immediate impact if it interferes with the patients' ability to maintain a normal lifestyle, additionally it is often perceived to have a substantial symbolic consequence in so far as it suggests persistent morbidity that is a reminder of the risk of death.

Fatigue is the most common adverse effect of chemotherapy, and it is a common complaint among women receiving hormonal and biological therapies for breast cancer. In many 
instances, however, there may be underlying factors, acute or chronic, that may be precipitating or exacerbating fatigue and tiredness. These may include anemia, infection, fever, dehydration, electrolyte imbalance, cachexia, hypothyroidism or hypogonadism, depression, sleep disturbance, and centrally acting sedating medications.

A rational treatment approach should be three pronged: ${ }^{33}$ first addressing underlying reversible factors, second using nonpharmacological, and finally pharmacological approaches.

Four nonpharmacological approaches are presented in Box 1.

Energy expenditure planning involved the planning of daily activities or prioritizing activities to be undertaken at times of leased fatigue and maximal energy. Energy restoration strategies include ensuring adequate rest and nutrition, reducing stress through techniques such as meditation or relaxation. Physical exercise has been endorsed as a useful approach in many clinical trials. ${ }^{34}$ The level of physical exercise needs to be tailored to the underlying performance status and general well-being of the patients.

Fatigue patients who are anemic with a hemoglobin of $<8 \mathrm{~g} / \mathrm{dL}$ can be considered for blood transfusion, ${ }^{35}$ aiming to maintain a hemoglobin level between 10 and $12 \mathrm{~g} / \mathrm{dL}$.

There are some limited data that patients with persistent fatigue may benefit from a low dose of either methylphenidate or modafanil. Methylphenidate is generally initiated at a dose of 5-10 $\mathrm{mg}$ in the morning and may be titrated up to 40-60 mg per day. ${ }^{33}$ The most common side effects with this approach include agitation, nervousness, sleep disturbances, nausea, or diarrhea.

Though steroids are not useful in the long-term management fatigue, they may have a role to play in the short-term management of fatigue for a patient who wants to have optimal alertness for an important occasion or family event.

Box I Nonpharmacological approaches to fatigue

\begin{tabular}{|l|l|}
\hline $\begin{array}{l}\text { Energy expenditure } \\
\text { planning }\end{array}$ & $\begin{array}{l}\text { Planning of daily activities or } \\
\text { prioritizing activities to be } \\
\text { undertaken at times of leased fatigue } \\
\text { and maximal energy }\end{array}$ \\
\hline Energy restoration strategies & $\begin{array}{l}\text { Ensuring adequate rest and nutrition, } \\
\text { reducing stress through techniques } \\
\text { such as meditation or relaxation }\end{array}$ \\
\hline Physical exercise & $\begin{array}{l}\text { Endorsed as a useful approach in } \\
\text { many clinical trials } \\
\text { The level of physical exercise needs } \\
\text { to be tailored to the underlying } \\
\text { performance status and general well- } \\
\text { being of the patients }\end{array}$ \\
\hline
\end{tabular}

In that situation, it is our common practice to administer dexamethasone $10-20 \mathrm{mg}$ at 12 hours before the event.

\section{Delirium and confusion}

Delirium can be described as alteration in attention and awareness fluctuating in severity across time. It is sometimes accompanied with neurological deficits such as aphasia, disorientation and hallucinations, and psychomotor changes. Delirium is a common complication of advanced cancer. Incidence estimates range from $43 \%$ in the general cancer population to $85 \%$ in patients in the terminal stages of their illness. ${ }^{36}$

Three clinical subtypes of delirium, based on arousal disturbance and psychomotor behavior, have been described as follows:

1. Hyperactive subtype - hyperaroused, hyperalert, or agitated.

2. Hypoactive subtype - hypoaroused, hypoalert, or lethargic.

3. Mixed subtype with alternating features of hyperactive and hypoactive delirium.

Neurologic abnormalities may be present during delirium, including cortical abnormalities (dysgraphia, constructional apraxia, and dysnomic aphasia); motor abnormalities (tremor, asterixis, myoclonus, and reflex or tone changes); and electroencephalogram abnormalities (typically global slowing).

The extent of evaluation is very much determined by the patients' prognosis. ${ }^{36}$ When delirium occurs in an ambulatory patient, it should generally be evaluated urgently. Comprehensive initial evaluation should be performed to identify and address the contributing factors, including obtaining the history with corroboration from family or staff, medication review, physical and neurological examination, and specific laboratory tests or imaging, as appropriate to the patients' goals of care.

Common precipitating events include sepsis, medication side effects, and metabolic aberrations (particularly hypercalcemia, hyponatremia, uremia, dehydration, brain metastases, or cerebrovascular events). ${ }^{36}$ Less common causes include leptomeningeal metastases or status epilepticus.

Delirium is a medical emergency that must be managed with compassion, reassurance, and clear explanation of the impact of pharmacological and nonpharmacological strategies to improve symptom control. ${ }^{36}$ Nonessential centrally acting medications that may contribute to delirium should be stopped.

Medications for treating agitation and delirium in the terminal phase include antipsychotics: the newer agents such 
as olanzapine, quetiapine, and aripiprazole appear less likely to be associated with extrapyramidal side effects than earlier generation antipsychotics such as haloperidol, chlorpromazine, levomepromazine, or risperidone. ${ }^{36}$ Benzodiazepines may be required for more rapid control of agitation and anxiety, but they can occasionally cause paradoxical agitation if used alone. ${ }^{36}$

\section{Evaluation and management of common psychological symptoms}

Breast cancer places considerable psychological stress on patients and their families. ${ }^{3}$ These stresses, similarly, amplify the emotional content of the contact with health care providers, particularly with oncologists. In order to be able to effectively care for cancer patients, oncology clinicians must be familiar with common psychological syndromes experienced by patients and their families.

\section{Anxiety}

Anxiety is endemic among patients with advanced breast cancer. ${ }^{37}$ Among patients, it commonly coexists with depressive symptoms, and it is very common among the family members of cancer patients. Anxiety has many expressions and identifiable themes that are related to their cancer experience $^{37}$ (Table 2).

In deciding whether to treat anxiety in a patient with cancer, the patient's subjective level of distress is the primary consideration for the initiation of treatment. Other considerations include problematic patient behaviors such as noncompliance due to anxiety, family and staff reactions to the patients' distress, and the balancing of the risks and benefits of treatment. Anxiolytic pharmacotherapy should usually be administered in conjunction with supportive psychotherapy or cognitive therapy approaches. ${ }^{39}$ The pharmacotherapy of anxiety in terminal illness typically involves use of benzodiazepine or nonbenzodiazepine anxiolytics. ${ }^{39}$

Benzodiazepines are the mainstay of the pharmacological treatment of anxiety in patients with cancer. The shorter acting benzodiazepines, such as lorazepam, alprazolam, and oxazepam, are the safest in this population. Longer acting benzodiazepines such as diazepam or clonazepam are usually reserved for patients who experience breakthrough anxiety or end of dose failure. Midazolam, a very short acting is useful in controlling anxiety and agitation in terminal phases of illness. For patients with persistent symptoms, neuroleptics such as olanzapine or quetiapine sometimes are indicated.

Nonpharmacological interventions include supportive psychotherapy and behavioral interventions that are used
Table 2 Common expressions and themes of anxiety and their clinical manifestations

\begin{tabular}{|l|l|}
\hline Expression of anxiety & $\begin{array}{l}\text { Common clinical } \\
\text { presentations }\end{array}$ \\
\hline Fearfulness & $\begin{array}{l}\text { Often characterized by } \\
\text { apprehension and dread } \\
\text { When severe, may become } \\
\text { even more exaggerated and } \\
\text { manifest as panic or alarm }\end{array}$ \\
\hline $\begin{array}{l}\text { Physical reactions not attributable to } \\
\text { organic disease or drug reactions }\end{array}$ & $\begin{array}{l}\text { Hyperventilation } \\
\text { Sensation of lump in throat } \\
\text { Palpitations }\end{array}$ \\
\hline Tightness in chest or stomach \\
Nausea
\end{tabular}

alone or in combination. Brief supportive psychotherapy, by a skilled therapist, is often useful in dealing with both crisis-related issues and existential issues confronted by the terminally ill. ${ }^{39}$ The goals of psychotherapy with the patient are to establish a bond that decreases the sense of isolation experienced with terminal illness; to help the patient face their illness and its treatment with a sense of self-worth; to correct misconceptions about the past and present; to integrate the present illness into a continuum of 
life experiences; and to explore issues of fears, loss, and the unknown that lies ahead. The therapist should emphasize past strengths and support previously successful ways of coping. This helps the patient mobilize inner resources, modify plans for the future, and perhaps even accept the inevitability of death.

The oncology clinicians can contribute greatly to alleviating anxiety by attending to the emotional and informational needs of patients and their families during the cancer experience and by ensuring open lines of communication and a commitment to rapid response in times of distress or concern.

\section{Depression}

Depression is less prevalent than anxiety. However, it is a common symptom and may well be underreported by patients. ${ }^{3,37}$ It is more common among patients with a family history of depression and history of previous depressive episodes. In some cases depression may be iatrogenic; and corticosteroids, hormonal therapies, and whole brain radiation have all been implicated as potential causes of depression.

Treating clinicians should be alert to common symptoms of depression (Table 3).

Depression in cancer patients often requires an approach combining supportive psychotherapy, cognitive-behavioral techniques, and antidepressant medications. ${ }^{40}$ Psychotherapy and cognitive-behavioral techniques -in the form of either individual or group counseling - are useful in the management of psychological distress in cancer patients and have been applied to the treatment of depressive and anxious symptoms related to cancer.

Table 3 Common symptoms of depression

\begin{tabular}{|l|l|}
\hline Symptoms of depression & Common clinical presentations \\
\hline Mood alteration & $\begin{array}{l}\text { Persistent sadness } \\
\text { Flatness of affect } \\
\text { Emotionally withdrawn and } \\
\text { unresponsive }\end{array}$ \\
\hline Ruminative negative thoughts & $\begin{array}{l}\text { Preoccupation with intrusive } \\
\text { thoughts about the illness and its } \\
\text { implications } \\
\text { Distracted and unable to focus on } \\
\text { other tasks at home or at work }\end{array}$ \\
\hline Passivity & $\begin{array}{l}\text { Disengagement from usual activities } \\
\text { and interests } \\
\text { Low motivation to do anything } \\
\text { Lack of interest in the other issues } \\
\text { going on in their lives }\end{array}$ \\
\hline Poor sleep and appetite & $\begin{array}{l}\text { Poor sleep and appetite unrelated } \\
\text { to the illness or its treatment. }\end{array}$ \\
\hline
\end{tabular}

Antidepressant pharmacotherapy is the mainstay of management in the treatment of cancer patients with severe depressive symptoms. ${ }^{40}$ This should almost always be accompanied by some form of psychotherapy by a skilled therapist. ${ }^{40,41}$ There are five selective serotonin re-uptake inhibitors including fluoxetine, sertraline, paroxetine, citalopram, and fluvoxamine. Fluoxetine and paroxetine may accelerate the metabolism of tamoxifen and are not preferred for patients on hormonal therapy. These drugs are as effective in the treatment of depression as the tricyclics but have a lower likelihood of major side effects. That is not to say that these drugs are free of adverse effects. Many patients complain of loose stools, nausea, vomiting, insomnia, headaches, and sexual dysfunction. Some patients may experience anxiety, tremor, and restlessness particularly when starting treatment. A third generation of antidepressants includes the serotonin-norepinephrine reuptake inhibitor, venlaflaxine, and the serotonin-2 antagonists/ serotonin reuptake inhibitors, nefazodone and trazodone. These agents share the improved therapeutic index of the SSRI drugs.

The role of tricyclic antidepressants is limited because of their anticholinergic side including constipation, dry mouth, and urinary retention. They may be a good option for the depressed patient who also suffers from agitation and insomnia. Psychostimulants such as methylphenidate are a useful alternative and effective approach to the treatment of depression particularly when a rapid onset of action is needed. ${ }^{41}$ Occasionally, treatment with SSRI and a psychostimulant may be initiated concurrently so that patients with depression may receive the immediate benefits of the psychostimulant drug until the 1-2 weeks necessary for an SSRI to begin to work pass. In relatively low dose, psychostimulants stimulate appetite, promote a sense of well-being, and improve feelings of weakness and fatigue in cancer patients.

\section{Existential suffering}

In addition to the well described psychological syndromes, existential issues are also common among patients with cancer. For patients with advanced cancer, these include concerns related to hopelessness, futility, meaninglessness, disappointment, remorse, death anxiety, and disruption of personal identity. ${ }^{42,43}$

Existential distresses may be related to past, present, or future concerns.

1. Present: Current personal integrity and identity can be disrupted by changes in body image; somatic, intellec- 
tual, social, and professional function and in perceived attractiveness as a person and as a sexual partner.

2. Past: For some patients, retrospection can trigger profound disappointment from unfulfilled aspirations or remorse from unresolved guilt or unsolved matters.

3. Future: If life is perceived to offer, at best, comfort in the setting of fading potency or, at worst, ongoing physical and emotional distress, anticipation of the future may be associated with feelings of hopelessness, futility, or meaninglessness such that the patients see no value in continuing to live.

Existential issues are universal and independent of religion and religious practice although their content is often influenced by one's culture. ${ }^{44}$ Patients with existential and spiritual distress may often be helped with skilled chaplaincy or spiritual care. Cognitive therapists have developed specific programs to enhance dignity ${ }^{45,46}$ and meaning. ${ }^{47,48}$

There is a place for the treating clinician to engage with some of these issues. ${ }^{49}$ For many patients, a lack of understanding about their illness and its treatment enhances feelings of vulnerability, uncertainty and helplessness. Often information is obfuscated with vague language with the intention of "protecting the patient" but this is often counterproductive. By sharing knowledge and information in a sensitive and appropriate manner, the treating clinician can often help to empower the patient to take command of their lives as much as possible. The discussions are often underscored by a wealth of feelings: anxiety, fear, sadness, and distress are all common. These discussions need to be accompanied by the opportunity to work through the emotional response, to prevent an emotional backlog which can undermine the process of coping. This requires that the treating clinicians acknowledge these feelings and allow the patients to ventilate them to release emotional energy that is essential in the process of re-establishing coping.

Even in the setting of advanced cancer, hope remains important. ${ }^{50}$ Clinicians can help the patients shift the focus to achievable and meaningful hope such as hope for time, hope for freedom from discomfort, hope to maximize the quality of one's life in the setting of threatened duration of survival, hope that one's loved ones will cope with the time ahead, hope that as one approaches the end of one's days that it will be with the feeling of satisfaction with the quality of what has been achieved, and hope for a peaceful death without suffering or indignity.

Talking about coping can be important and helpful. ${ }^{3}$ To some degree, the challenge of living and coping with cancer is related to attitude: giving all of the uncertainties associated with the disease patients can see themselves as "living with cancer" or as “dying of cancer." Some patients develop maladaptive responses to their illness that undermine the efficacy of their coping. Some "resign and withdraw" with a progressive and biologically premature invalidity which, in itself, may alienate their families and care givers. Some "Reject and Rage" 51,52 expressing their anger as if they are trying to make everyone around them as miserable as possible. Maladaptive coping needs to be recognized and addressed by the care team to minimize both patient and family distress.

\section{Common challenging palliative care problems in breast cancer}

\section{Fungating chest wall tumors}

In many instances, patients initially presenting with ulcerated or fungating chest wall tumors will respond to anticancer therapies. ${ }^{53}$ In some situations, especially with initial presentation, anti-tumor therapies (hormonal, chemotherapy, and biological) or radiation therapy also enhance local control.

Persistent chest wall and skin lesions after several lines of therapy present major treatment challenges. ${ }^{53}$ These lesions may be complicated by problems of itch, pain exudates, infection, and odor ${ }^{54}$ and are a major cause of distress to patients and their caregivers. ${ }^{54}$ These patients need meticulous wound care, adequate supports, and analgesia particularly for wound care and open lines of communication with the professional care team.

For patients with good performance status and a life expectancy of more than 6 months, surgical management with resection with appropriate flap or graft reconstruction is occasionally helpful, ${ }^{55}$ but for most patients this will not be an option. These patients and their caregivers need education and support to assist with washing, asepsis, dressing changes, control of odor, itch and bleeding, pain management, and dealing with the most troublesome chronic wound problems that affect the patients physically and emotionally. ${ }^{56,57}$

Initial management includes debridement and proper wound dressings. Nonadherent dressings should be used to reduce bleeding and pain with dressing changes, that is, special dressings such as foams, alginates, or starch copolymers which reduce the need for frequent dressing changes. Odor can be controlled with interval mechanical debridement to decrease the microbial bioburden on the wound surface and dressings that incorporate topical metronidazole, hydro foam with silvers sulfadiazine, or medicinal honey ${ }^{58}$ When there is oozing from the lesions, topical hemostatic agents such as gelatin (Gelfoam) or collagen (Helistat) can be applied. Other approaches that have been described include gauze saturated with adrenaline or sucralfate paste $(1 \mathrm{~g}$ sucralfate tablet in 5 $\mathrm{mL}$ of water-soluble gel) may decrease widespread oozing. ${ }^{57,59}$ 
The management plan should be considerate of esthetics including attention to dressings that protect clothing from discharge and that preserve symmetry as much as possible.

Isolated bleeding points may be treated with silver nitrate sticks; however, more active bleeding may necessitate more aggressive approaches including transcatheter embolization of bleeding arteries ${ }^{59,60}$ and direct puncture sclerotherapy in which the tumor is injected with sclerosing agent, radiotherapy, or rarely reconstructive surgery. ${ }^{59}$ Keeping the skin moisturized and protected will help reduce itching, and, if necessary, topical corticosteroid creams can be applied.

Chronic pain should be treated in accordance with the standard guidelines for chronic cancer pain. Dressing changes will often require premedication with a short-acting opioids and, occasionally, may even require sedation.

\section{Brain metastases}

Brain metastases are a common complication of breast cancer which occurs in $\sim 15 \%$ of patients with metastatic breast cancer. ${ }^{61}$ The risk of breast brain metastases is very low among patients with luminal A breast cancer and is substantially higher among patients with luminal B, Her2-positive, and triple-negative breast cancer. ${ }^{61,62}$

Common symptoms associated with brain metastases include headache, confusion, and new onset of focal seizures. Diagnosis is made through axial brain imaging using either contrast-enhanced CT scan or gadolinium enhanced MRI scanning. ${ }^{63}$ Given the more limited diagnostic resolution of CT scanning, when a small number of brain metastases are identified that may be amenable to localized therapies, a subsequent MRI scan is indicated for more accurate staging of the true extent of cerebral metastases. ${ }^{63}$

Symptomatic patients with extensive vasogenic edema axial imaging should be treated with dexamethasone. ${ }^{63}$ Moderate- to high-dose steroids can cause a reduction in intracranial pressure and reduction in symptoms within hours of administration. A starting dose of 10-20 mg can be used. This can be gradually tapered down after a clinical improvement is achieved. Anticonvulsant therapies are indicated for patients who have had a seizure associated with their metastasis. Routine seizure prophylaxis is not recommended.

Among patients with a relatively well preserved performance status, the treatment strategies are influenced by the number of involved metastases. When there is a single lesion, this is best managed with the surgical resection if this is technically possible. ${ }^{63}$ Postoperative radiotherapy ought to be considered. There is increasing data to indicate that the problems of cognitive decline may be reduced by using more targeted approaches to adjuvant radiotherapy including hippocampal sparing radiotherapy or stereotactic surgery to the resection cavity.

Stereotactic radiotherapy (sometimes called radiosurgery or gamma knife surgery) is recommended when there is a small number of brain metastases (usually $<5$ ) and for patients who have a single metastasis but who are not candidates for surgery. ${ }^{63}$ There is no evidence that subsequent brain radiotherapy reduces the risk of recurrence or improves overall survival.

Whole brain radiotherapy may be appropriate for symptomatic patients who have a very poor prognosis and for patients who have multiple metastases not amenable to focal therapy. ${ }^{63,64}$ For patients with HER2 overexpressing breast cancer, the trial of therapy with lapatinib and capecitabine may provide adequate control and allow for the deferral of whole brain radiotherapy. ${ }^{64,65}$

In the setting of recurrent disease, patients with a good performance status and a limited number of recurrent lesions may be treated with stereotactic radiosurgery even if they have previously received whole brain radiotherapy. ${ }^{63,66}$

\section{Leptomeningeal metastases}

Leptomeningeal metastases that are characterized by diffuse or multifocal involvement of the subarachnoid space by metastatic tumor occur in a small proportion of patients with breast cancer usually late in the evolution of the disease. ${ }^{67,68}$ This is a poor prognostic situation with a median overall survival of $<4$ months. ${ }^{69}$

Leptomeningeal metastases present with focal or multifocal neurological symptoms or signs that may involve any level of the neuraxis. ${ }^{67,68}$ The most common presenting symptoms are headache, cranial nerve palsies, ${ }^{70}$ and radicular pain in the low back and buttocks. ${ }^{71}$ More than one-third of patients presents with evidence of cranial nerve damage, including double vision, hearing loss, facial numbness, and decreased vision. ${ }^{70}$

Gadolinium-enhanced MRI of the neuroaxis is the investigation of choice when leptomeningeal metastases are suspected. Compared to cerebrospinal fluid (CSF) cytology examination, MRI probably is more sensitive but is less specific as false-positive cytologies are rare. ${ }^{72}$ Gadoliniumenhanced spinal MRI may be positive in almost $50 \%$ of patients without clinical findings related to the spinal region and in $60 \%$ of patients with negative CSF cytology. ${ }^{73}$ The CSF analysis may reveal elevated pressure, elevated protein, depressed glucose, and/or lymphocytic pleocytosis. Ninety percent of patients ultimately show positive cytology, but multiple evaluations may be required. Despite these measures, 
CSF cytology is persistently negative in as many as $20 \%$ of patients with clinically or radiographically unequivocal leptomeningeal involvement.

Patients with poor performance status, multiple fixed neurologic deficits, and extensive systemic cancer with limited therapeutic options have a poor prognosis, and there is little likelihood of benefit from primary therapies in this setting and symptomatic care is recommended..$^{67,68}$ In addition to analgesics, corticosteroids may improve headache and radicular pain. Anticonvulsants should be used for patients with seizures. Occasionally, ventriculoperitoneal shunting can be indicated for the management of obstructive hydrocephalus causing intractable headache, encephalopathy, and incontinence.

For patients with a good performance status, no or modest fixed neurologic deficits, and minimal systemic disease burden, a more active approach is justifiable.$^{67,68}$ This may include management of raised intracranial pressure with corticosteroids; CSF flow study (radionuclide cisternogram) to define areas of obstruction to CSF flow, focal radiotherapy for the initial management of areas of bulky or symptomatic disease, and for the treatment of any obstructions to CSF flow. There is insufficient evidence to endorse routine use of intrathecal methotrexate or thiotepa or high-dose systemic methotrexate..$^{67,68}$

Use of intrathecal trastuzumab in patients with HER2+ breast cancer has been described in case reports, with cytologic and radiographic responses and few cases of prolonged response. ${ }^{74-76}$

\section{Conclusion}

Patients with advanced breast cancer and their families have complex needs, which, when unmet, can result in severe distress and undermine their quality of life. Optimizing the quality of life and well-being of these patients and their families requires a meticulous approach to supportive and palliative care. This task requires the full engagement of an interdisciplinary approach to palliative care with strong emphasis on the assessment of needs and anticipated needs, skilled therapeutics, and a commitment to continuity of care.

\section{Disclosure}

The authors report no conflicts of interest in this work.

\section{References}

1. Mariotto AB, Etzioni R, Hurlbert M, Penberthy L, Mayer M. Estimation of the Number of Women Living with Metastatic Breast Cancer in the United States. Cancer Epidemiol Biomarkers Prev. 2017;26(6):809-815.

2. Thrift-Perry M, Cabanes A, Cardoso F, Hunt KM, Cruz TA, Faircloth K. Global analysis of metastatic breast cancer policy gaps and advocacy efforts across the patient journey. Breast. 2018;41:93-106.
3. Brunault P, Champagne AL, Huguet G. Major depressive disorder, personality disorders, and coping strategies are independent risk factors for lower quality of life in non-metastatic breast cancer patients. Psycho-Oncology. 2016;25:513-520.

4. Raskin W, Harle I, Hopman WM, Booth CM. Prognosis, Treatment Benefit and Goals of Care: What do Oncologists Discuss with Patients who have Incurable Cancer? Clin Oncol ( $R$ Coll Radiol). 2016;28(3):209-214.

5. Greer JA, Jackson VA, Meier DE, Temel JS. Early integration of palliative care services with standard oncology care for patients with advanced cancer. CA Cancer J Clin. 2013;63(5):349-363.

6. Meyers FJ, Linder J, Beckett L, Christensen S, Blais J, Gandara DR. Simultaneous care: a model approach to the perceived conflict between investigational therapy and palliative care. J Pain Symptom Manage. 2004;28(6):548-556.

7. Bakitas M, Lyons KD, Hegel MT, et al. Effects of a palliative care intervention on clinical outcomes in patients with advanced cancer: the Project ENABLE II randomized controlled trial. JAMA. 2009;302(7):741-749.

8. Temel JS, Greer JA, Muzikansky A, et al. Early palliative care for patients with metastatic non-small-cell lung cancer. $N$ Engl J Med. 2010;363(8):733-742.

9. Zimmermann C, Swami N, Rodin G, et al. Cluster-randomized trial of early palliative care for patients with metastatic cancer. Presented at: American Society for Clinical Oncology Annual Meeting; 2012; Chicago.

10. Gade G, Venohr I, Conner D, et al. Impact of an inpatient palliative care team: a randomized control trial. J Palliat Med. 2008;11(2):180-190.

11. Brumley R, Enguidanos S, Jamison P, et al. Increased satisfaction with care and lower costs: results of a randomized trial of in-home palliative care. J Am Geriatr Soc. 2007;55(7):993-1000.

12. Jordhøy MS, Fayers P, Loge JH, Ahlner-Elmqvist M, Kaasa S. Quality of life in palliative cancer care: results from a cluster randomized trial. J Clin Oncol. 2001;19(18):3884-3894.

13. Ahmed N, Ahmedzai SH, Collins K. Holistic assessment of supportive and palliative care needs: the evidence for routine systematic questioning. BMJ Support Palliat Care. 2014;4(3):238-246.

14. Hallenbeck J, Arnold R. A request for nondisclosure: don't tell mother. J Clin Oncol. 2007;25(31):5030-5034.

15. Ali SK. Malakouti M: The family meeting. Palliat Support Care. 2015;13(4):1135-1136.

16. Nenner F. Goals of care: a family meeting. J Palliat Med. 2013;16(1):103-104.

17. Cleeland CS, Gonin R, Hatfield AK, et al. Pain and its treatment in outpatients with metastatic cancer. N Engl J Med. 1994;330:592-596.

18. BT Li, Wong MH, Pavlakis N. Treatment and prevention of bone metastases from breast cancer: a comprehensive review of evidence for clinical practice. J Clin Med. 2014;3(1):1-24.

19. Mercadante S, Fulfaro F. Management of painful bone metastases. Curr Opin Oncol. 2007;19(4):308-314.

20. Singh PC, Patel DV, Chang VT. Metastatic acetabular fractures: evaluation and approach to management. J Pain Symptom Manage. 2006;32(5):502-507.

21. Ripamonti CL, Santini D, Maranzano E, Berti M, Roila F; ESMO Guidelines Working Group. Management of cancer pain: ESMO clinical practice guidelines. Ann Oncol. 2012;23:vii139-vii154.

22. Ropper AE, Ropper AH. Acute Spinal Cord Compression. $N$ Engl J Med. 2017;376(14):1358-1369.

23. George R, Jeba J, Ramkumar G, Chacko AG, Tharyan P. Interventions for the treatment of metastatic extradural spinal cord compression in adults. Cochrane Database Syst Rev. 2015;(9):CD006716.

24. Al-Qurainy R, Collis E. Metastatic spinal cord compression: diagnosis and management. BMJ. 2016;353:i2539.

25. Rades D, Huttenlocher S, Dunst J, et al. Matched pair analysis comparing surgery followed by radiotherapy and radiotherapy alone for metastatic spinal cord compression. J Clin Oncol. 2010;28(22):3597-3604.

26. Ilhan E, Chee E, Hush J, Moloney N. The prevalence of neuropathic pain is high after treatment for breast cancer: a systematic review. Pain. 2017;158(11):2082-2091. 
27. Niravath P. Aromatase inhibitor-induced arthralgia: a review. Ann Oncol. 2013;24(6):1443-1449.

28. Tofthagen C, McAllister RD, Visovsky C. Peripheral neuropathy caused by Paclitaxel and docetaxel: an evaluation and comparison of symptoms. J Adv Pract Oncol. 2013;4(4):204-215.

29. Shimozuma K, Ohashi Y, Takeuchi A, et al. Taxane-induced peripheral neuropathy and health-related quality of life in postoperative breast cancer patients undergoing adjuvant chemotherapy: N-SAS BC 02, a randomized clinical trial. Support Care Cancer. 2012;20(12):3355-3364.

30. Alleaume C, Bendiane MK, Bouhnik AD, et al. Chronic neuropathic pain negatively associated with employment retention of cancer survivors: evidence from a national French survey. J Cancer Surviv. 2018;12(1):115-126.

31. Finnerup NB, Attal N, Haroutounian S, et al. Pharmacotherapy for neuropathic pain in adults: a systematic review and meta-analysis. Lancet Neurol. 2015;14(2):162-173.

32. Kloke M, Cherny N. Treatment of dyspnoea in advanced cancer patients: ESMO Clinical Practice Guidelines. Ann Oncol. 2015;26(Suppl 5): v169-v173.

33. Mohandas H, Jaganathan SK, Mani MP, Ayyar M, Rohini Thevi GV. Cancer-related fatigue treatment: An overview. $J$ Cancer Res Ther. 2017;13(6):916-929.

34. Juvet LK, Thune I, Elvsaas IKØ, et al. The effect of exercise on fatigue and physical functioning in breast cancer patients during and after treatment and at 6 months follow-up: A meta-analysis. Breast. 2017;33:166-177.

35. Carson JL, Guyatt G, Heddle NM, et al. Clinical practice guidelines from the AABB: red blood cell transfusion thresholds and storage. JAMA. 2016;316(19):2025-2035.

36. Bush SH, Lawlor PG, Ryan K, et al. Delirium in adult cancer patients: ESMO Clinical Practice Guidelines. Ann Oncol. 2018;29 (Suppl 4):iv143-iv165.

37. Park EM, Gelber S, Rosenberg SM, et al. Anxiety and Depression in Young Women With Metastatic Breast Cancer: A Cross-Sectional Study. Psychosomatics. 2018;59(3):251-258.

38. Dinkel A, Herschbach P. Fear of Progression in Cancer Patients and Survivors. Recent Results Cancer Res. 2018;210:13-33.

39. Roth AJ, Massie MJ. Anxiety and its management in advanced cancer. Curr Opin Support Palliat Care. 2007;1(1):50-56.

40. Rodin G. Effective treatment for depression in patients with cancer. Lancet. 2014;384(9948):1076-1078.

41. Li M, Kennedy E, Byrne N. The Management of Depression in Patients with Cancer. Toronto: Cancer Care Ontario; 2015.

42. Boston P, Bruce A, Schreiber R. Existential suffering in the palliative care setting: an integrated literature review. J Pain Symptom Manage. 2011;41(3):604-618.

43. Kissane DW. The relief of existential suffering. Arch Intern Med. 2012;172(19):1501-1505.

44. Puchalski CM, King SDW, Ferrell BR. Spiritual Considerations. Hematol Oncol Clin North Am. 2018;32(3):505-517.

45. Chochinov HM, Hack T, Hassard T, Kristjanson LJ, McClement S, Harlos M. Dignity therapy: a novel psychotherapeutic intervention for patients near the end of life. J Clin Oncol. 2005;23(24):5520-5525.

46. Martinez M, Arantzamendi M, Belar A, et al. "Dignity therapy", a promising intervention in palliative care: A comprehensive systematic literature review. Palliat Med. 2017;31(6):492-509.

47. Breitbart W, Pessin H, Rosenfeld B, et al. Individual meaning-centered psychotherapy for the treatment of psychological and existential distress: A randomized controlled trial in patients with advanced cancer. Cancer. 2018;124(15):3231-3239.

48. Breitbart W, Rosenfeld B, Pessin H, Applebaum A, Kulikowski J, Lichtenthal WG. Meaning-centered group psychotherapy: an effective intervention for improving psychological well-being in patients with advanced cancer. J Clin Oncol. 2015;33(7):749-754.

49. Rodin D, Balboni M, Mitchell C, Smith PT, VanderWeele TJ, Balboni TA. Whose role? Oncol practitioners' perceptions of their role in providing spiritual care to advanced cancer patients. Support Care Cancer. 2015;23(9):2543-2550.
50. McClement SE, Chochinov HM. Hope in advanced cancer patients. Eur J Cancer. 2008;44(8):1169-1174.

51. Gerhart J, Schmidt E, Lillis T, O’Mahony S, Duberstein P, Hoerger M. Anger Proneness and Prognostic Pessimism in Men With Prostate Cancer. Am J Hosp Palliat Care. 2017;34(6):497-504.

52. O'Grady E, Dempsey L, Fabby C. Anger: a common form of psychological distress among patients at the end of life. Int $J$ Palliat Nurs. 2012;18(12):592-596.

53. Alexander S. Malignant fungating wounds: epidemiology, aetiology, presentation and assessment. JWound Care. 2009;18(7):273-4, 276-8, 280 .

54. Alexander S. Malignant fungating wounds: key symptoms and psychosocial. J Wound Care. 2009;18(12):325-329.

55. Petrella F, Radice D, Borri A, et al. Chest wall resection and reconstruction for locally recurrent breast cancer: From technical aspects to biological assessment. Surgeon. 2016;14(1):26-32.

56. Merz T, Klein C, Uebach B, Kern M, Ostgathe C, Bükki J. Fungating wounds-multidimensional challenge in palliative care. Breast Care (Basel). 2011;6(1):21-24

57. Seaman S. Management of malignant fungating wounds in advanced cancer. Semin Oncol Nurs. 2006;22(3):185-193.

58. Lund-Nielsen B, Adamsen L, Kolmos HJ, Rørth M, Tolver A, Gottrup F. The effect of honey-coated bandages compared with silver-coated bandages on treatment of malignant wounds - a randomized study. Wound Repair Regen. 2011;19(6):664-670.

59. Pereira J, Phan T. Management of bleeding in patients with advanced cancer. Oncologist. 2004;9(5):561-570.

60. Aksoy Ş, Akçe B, Kılıçkesmez Ö, et al: Transcatheter Arterial Embolization for Controlling Severe Bleeding From Recurrent Locally-Advanced Breast Cancer. J Breast Health. 2016;12:137-140.

61. Frisk G, Tinge B, Ekberg S, et al. Survival and level of care among breast cancer patients with brain metastases treated with whole brain radiotherapy. Breast Cancer Res Treat. 2017;166(3):887-896.

62. Arvold ND, Oh KS, Niemierko A, et al. Brain metastases after breastconserving therapy and systemic therapy: incidence and characteristics by biologic subtype. Breast Cancer Res Treat. 2012;136(1):153-160.

63. Soffietti R, Abacioglu U, Baumert B, et al. Diagnosis and treatment of brain metastases from solid tumors: guidelines from the European Association of Neuro-Oncology (EANO). Neuro Oncol. 2017;19(2):162-174.

64. O’Sullivan CC, Davarpanah NN, Abraham J, Bates SE. Current challenges in the management of breast cancer brain metastases. Semin Oncol. 2017;44(2):85-100.

65. Bachelot T, Romieu G, Campone M, et al. Lapatinib plus capecitabine in patients with previously untreated brain metastases from HER2-positive metastatic breast cancer (LANDSCAPE): a single-group phase 2 study. Lancet Oncol. 2013;14(1):64-71.

66. Kelly PJ, Lin NU, Claus EB, et al. Salvage stereotactic radiosurgery for breast cancer brain metastases: outcomes and prognostic factors. Cancer. 2012;118:2014-2020.

67. Scott BJ, Oberheim-Bush NA, Kesari S. Leptomeningeal metastasis in breast cancer - a systematic review. Oncotarget. 2016;7(4):3740-3747.

68. Scott BJ, Kesari S. Leptomeningeal metastases in breast cancer. Am J Cancer Res. 2013;3(2):117-126.

69. Morikawa A, Jordan L, Rozner R, et al. Characteristics and Outcomes of Patients With Breast Cancer With Leptomeningeal Metastasis. Clin Breast Cancer. 2017;17(1):23-28.

70. Yamanaka R, Koga H, Yamamoto Y, Yamada S, Sano T, Fukushige T. Characteristics of patients with brain metastases from lung cancer in a palliative care center. Support Care Cancer. 2010;19(4):467-73.

71. van Oostenbrugge RJ, Twijnstra A. Presenting features and value of diagnostic procedures in leptomeningeal metastases. Neurology. 1999;53(2):382-385.

72. Straathof CS, de Bruin HG, Dippel DW, Vecht CJ. The diagnostic accuracy of magnetic resonance imaging and cerebrospinal fluid cytology in leptomeningeal metastasis. J Neurol. 1999;246(9):810-814.

73. Gomori JM, Heching N, Siegal T. Leptomeningeal metastases: evaluation by gadolinium enhanced spinal magnetic resonance imaging. $J$ Neurooncol. 1998;36(1):55-60. 
74. Bousquet G, Darrouzain F, de Bazelaire C, et al. Intrathecal Trastuzumab Halts Progression of CNS Metastases in Breast Cancer. J Clin Oncol. 2016;34(16):e151-e155.

75. Pluchart H, Jacquet E, Charlety D, Allenet B, Bedouch P, Mousseau M. Long-Term Survivor with Intrathecal and Intravenous Trastuzumab Treatment in Metastatic Breast Cancer. Target Oncol. 2016;11(5):687-691.
76. Figura NB, Long W, Yu M, et al. Intrathecal trastuzumab in the management of HER2+ breast leptomeningeal disease: a single institution experience. Breast Cancer Res Treat. 2018;169(2):391-396.
Breast Cancer - Targets and Therapy

\section{Publish your work in this journal}

Breast Cancer - Targets and Therapy is an international, peerreviewed open access journal focusing on breast cancer research, identification of therapeutic targets and the optimal use of preventative and integrated treatment interventions to achieve improved outcomes, enhanced survival and quality of life for the cancer patient

\section{Dovepress}

The manuscript management system is completely online and includes a very quick and fair peer-review system, which is all easy to use. Visit http://www.dovepress.com/testimonials.php to read real quotes from published authors.

Submit your manuscript here: https:/www.dovepress.com/breast-cancer---targets-and-therapy-journal 